\title{
Bài và tác giả ĐH Phenikaa trên các ấn phẩm có HSTĐ vượt trội
}

\author{
Hồ Mạnh Toàn \\ Trung tâm ISR \\ Trường Đại học Phenikaa
}

Phenikaa Uni | ISR (2-7-2020; URL: https://isr.phenikaa-uni.edu.vn/chitiet/tin-

tuc 2019222758 84/bai-va-tac-gia-dh-phenikaa-tren-cac-an-pham-co-hstd-vuot-troi) - Bên cạnh khuôn viên và diện mạo mới khang trang hiện nay, Trường ĐH Phenikaa cũng trong quá trình tích cực lao động khoa học. Trong đó, không thể không nhắc tới lực lượng giảng viên đang say mê nghiên cứu, đóng góp cho công bố quốc tế (CBQT) của trường. Riêng nửa đầu 2020, Phenikaa Uni đã đóng góp 125 CBQT (Phenikaa-Uni, 2020).

Tuy nhiên, trong hoạt động khoa học, số lượng không phải là yếu tố quyết định. Uy tín khoa học còn bắt buộc phải được gây dựng qua những bài CBQT tốt. Một trong những chỉ dấu quan trọng bậc nhất chính là việc xuất bản được trên các ấn phẩm có hệ số tác động cao (HSTĐ).

Trong thế giới hàn lâm, HSTĐ được quan tâm nhất và có uy tín cao nhất là Journal Impact Factor (JIF) của Institute of Scientific Information, một số đo thống kê trích dẫn do người sáng lập ISI Eugene Garfield phát kiến. Ngày nay, JIF do Web of Science Group (thuộc Clarivate Analytics) xuất bản vào cuối tháng 6 hàng năm, trong một ấn phẩm được cả thế giới khoa học mong chờ Journal Citation Reports (JCR). Những ai quan tâm tới tình hình biến động HSTĐ, cũng như các ý kiến xung quanh JIF-còn hay được viết tắt là IF-có thể tham khảo bài của Trung tâm ISR xuất bản gần đây (Tran et al., 2020). Qua bài này, ta có thể thấy số lượng tạp chí có $\mathrm{IF} \geq 10$ là con số rất nhỏ trong hơn 20.000 ấn phẩm có IF do ISI Web of Science chỉ mục hóa. Con số đó chỉ xấp xỉ 260, chiếm chừng $2.2 \%$ tổng số ấn phẩm.

Journal Citation Reports 2020 được công bố ngày 29-6-2020 vừa qua (Collier, 2020), và dữ liệu đó là cảm hứng cho các phân tích nhanh sau đây.

\section{Dữ liệu phục vụ phân tích}

Bài sử dụng dữ liệu từ hai nguồn chính:

- Thống kê về CBQT của Phenikaa-Uni, có thể tìm đọc tại địa chỉ URL: https://phenikaauni.edu.vn/chitiet/tin-tieu-bieu/danh-sach-bai-bao-isi-scopus.

- Dữ liệu JIF của từng tạp chí qua Journal Citation Reports 2020.

Từ hai nguồn dữ liệu này, tác giả tiến hành lọc ra các bài CBQT và các tác giả có địa chỉ từ Phenikaa University. Một tiêu chí đặc biệt quan trọng là các bài phải được đăng trên các ấn phẩm có IF $\geq 10$ tương ứng với $J C R$ của năm xuất bản bài báo. Nghĩa là nếu bài xuất bản năm 2019, thì tiêu chuẩn lọc IF lấy từ báo cáo JCR 2020 của ISI Web of Science (do JCR 2020 sẽ cung cấp JIF 2019). Riêng các bài xuất bản nửa đầu 2020, thì sử dụng JCR 2020, là số liệu gần nhất có thể có.

Sau đó, số được lọc này được sắp xếp theo các tiêu chí khác nhau để rút ra quan sát. 
*Ghi chú: Do sử dụng các dữ liệu đã công khai trên trang web và chỉ đến thời điểm cuối tháng 6-2020, phân tích có thể bỏ sót các CBQT mới mà chưa được cập nhật. Nếu phát hiện được thiếu sót, xin vui lòng cung cấp thông tin để bài viết cập nhật. Liên lạc: toan.homanh@phenikaa-uni.edu.vn

\section{Một số quan sát rút ra từ dữ liệu CBQT}

Dữ liệu có được cho phép xây dựng một số bảng được trình bày dưới đây, giúp chúng ta thu nhận được một số quan sát tổng thể lý thú.

Trước tiên là bảng 1 cho thấy các tác giả Phenikaa Uni đóng góp trên các ấn phẩm HSTĐ cao vượt trội trong giai đoạn 2018-2020.

Bảng 1. Thời gian, và vài thông số liên quan tới ấn phẩm IF $\geq 10$

\begin{tabular}{|l|c|c|c|c|}
\hline Tác giả & Năm & $\begin{array}{c}\text { HSTÐ (IF) cao } \\
\text { nhất }\end{array}$ & $\begin{array}{c}\text { Số bài trên ấn } \\
\text { phẩm IF } \geq 10\end{array}$ & $\begin{array}{c}\text { Số ấn phẩm } \\
\mathrm{IF} \geq 10\end{array}$ \\
\hline Vương Quân Hoàng & $2018,2019,2020$ & 42.778 & 3 & 2 \\
\hline Nguyễn Công Lượng & 2020 & 23.700 & 2 & 2 \\
\hline Nguyễn Văn Hiếu & 2019 & 16.602 & 2 & 1 \\
\hline Vũ Ngọc Hùng & 2020 & 16.602 & 1 & 1 \\
\hline Đào Văn Dương & 2020 & 16.602 & 1 & 1 \\
\hline Phạm Thành Huy & 2019 & 12.300 & 1 & 1 \\
\hline Raja Das & 2019 & 12.300 & 1 & 1 \\
\hline Dương Anh Tuấn & 2019 & 12.300 & 1 & 1 \\
\hline
\end{tabular}

Điều đáng nói là, chỉ từ khi mang tên Phenikaa Uni vào năm 2018, trường mới bắt đầu ghi nhận có bài trên ấn phẩm HSTĐ cao. Và kể từ 2018, liên tục có các bài trên những ấn phẩm theo tiêu chí này. Có người liên tục 3 năm có đóng góp. Lại có tác giả năm 2020 là lần đầu tiên đóng góp, nhưng ngay lập tức đã có tới 2 bài trên 2 ấn phẩm HSTĐ cao ra đời trong nửa đầu 2020 .

Tiếp theo, chúng ta nhìn vào phân bố số bài, tác giả và ấn phẩm theo 4 mức hệ số tác động của khoảng cao từ IF 10 trở lên như trong Bảng 2.

Bảng 2. Phân bố số bài, tác giả, ấn phẩm theo các khoảng HSTĐ cao

\begin{tabular}{|l|c|c|c|c|}
\hline JIF & $10-19.999$ & $20-29.999$ & $30-39.999$ & $\geq 40$ \\
\hline Số bài & 7 & 1 & 0 & 1 \\
\hline Số tác giả & 8 & 1 & 0 & 1 \\
\hline Số ấn phẩm & 4 & 1 & 0 & 1 \\
\hline
\end{tabular}

Dữ liệu CBQT của Phenikaa Uni cho biết chi tiết về các tạp chí IF cao vượt trội đã đăng bài của các tác giả Phenikaa Uni bao gồm:

1. Nature $(2020,42.778)$

2. IEEE Communications Surveys and Tutorials $(2020 ; 23.700)$ 
3. Nano Energy (2019-2020; 16.602)

4. Materials Horizons $(2019 ; 12.300)$

5. IEEE Communications Magazine $(2020 ; 11.052)$

6. Nature Human Behaviour (2018-2019; 10.575-12.282)

Cũng theo thời gian, số lượng các tác giả của Phenikaa Uni tham gia xuất bản bài trên các tạp chí HSTĐ cao tăng dần: 1 (2018); 5 (2019); 4 (nửa đầu 2020).

Để sản xuất các bài này, các tác giả Phenikaa Uni cũng có phương thức hoạt động hợp tác đa dạng, từ solo tới tập thể. Bài có nhiều tác giả tham gia nhất là 13 tác giả (cũng chưa phải là nhiều trong khoa học hiện đại, nhưng cũng cho thấy khả năng hợp tác đa dạng, phong phú).

\section{Thay cho kết luận}

Phân tích này thể hiện một góc nhìn bổ sung cho thông tin về kết quả CBQT của Trường ĐH Phenikaa. Việc có thể xuất bản trên các ấn phẩm có HSTĐ rất cao như vừa phân tích là công việc vô cùng gian lao và thách thức. Tuy nhiên, có thể thấy một văn hóa CBQT chấp nhận khó khăn thách thức bậc nhất đã và đang phát triển tại Phenikaa Uni, với số CBQT trên ấn phẩm HSTĐ cao vượt trội đang tăng lên và số lượng tác giả cũng tăng lên.

Cùng với Nature Index Ranking, thông tin về CBQT trên các tạp chí 'high impact-factor' sẽ góp phần tạo dựng hình ảnh và uy tín khoa học của Phenikaa Uni trong hệ thống nghiên cứu quốc tế.

\section{Tài liệu tham khảo:}

Collier, K. (2020, June 29). The 2020 Journal Citation Reports: Helping you make better-informed decisions with confidence. Web of Science Group. Available from:

https://clarivate.com/webofsciencegroup/article/the-2020-journal-citation-reports-helping-youmake-better-informed-decisions-with-confidence/ (accessed: July 2, 2020)

Dao, V. D., Vu, N. H., \& Yun, S. (2020). Recent advances and challenges for solar-driven water evaporation system toward applications. Nano Energy, 68, 104324.

Geng, R., Luong, H. M., Pham, M. T., Das, R., Repa, K. S., Robles-Garcia, J., ... \& Larsen, G. K. (2019). Magnetically tunable organic semiconductors with superparamagnetic nanoparticles. Materials Horizons, 6(9), 1913-1922.

Ippili, S., Jella, V., Eom, J.-H., Kim, J., Hong, S., Tran, V.-D., Hieu, N. V. , \& Yoon, S.-G. (2019). An Ecofriendly flexible piezoelectric energy harverter that delivers high output performance is based on lead-free MASnl3 Films and MASnI3-PVDF composite films. Nano Energy, 57, 911-923.

Jella, V., Ippili, S., Eom, J.-H., Pammi, S. V. N., Jung, H.-S., Tran, V.-D., Hieu, N.-V., Kirakosyan, A., Kim, D., Sihn, M. R., Choi, J., Kim, Y.-J., \& Yoon, S.-G. (2019). A comprehensive review of flexible 
piezoelectirc generator based on organic-inorganic metal halide perovskites. Nano Energy, 57, 74-93.

Lim, W. Y. B., Luong, N. C., Hoang, D. T., Jiao, Y., Liang, Y. C., Yang, Q., ... \& Miao, C. (2020). Federated learning in mobile edge networks: A comprehensive survey. IEEE Communications Surveys \& Tutorials. (Article in Press)

Luong, N. C., Jiao, Y., Wang, P., Niyato, D., Kim, D. I., \& Han, Z. (2020). A Machine-Learning-Based Auction for Resource Trading in Fog Computing. IEEE Communications Magazine, 58(3), 82-88.

Phenikaa-Uni. (2020). Bài báo ISI/Scopus. Available from: https://phenikaa-uni.edu.vn/chitiet/tin-tieubieu/danh-sach-bai-bao-isi-scopus (access: July 2, 2020)

Tran, T., Hoang, L.-K., La, V.-P., Ho, M.-T., \& Vuong, Q. H. (2020). Scrambling for higher metrics in the Journal Impact Factor bubble period: a real-world problem in science management and its implications. Problems and Perspectives in Management, 18(1), 48-56.

Vuong, Q.-H. (2018). The (ir)rational consideration of the cost of science in transition economies. Nature Human Behaviour, 2(1), 5.

Vuong, Q.-H. (2019). Breaking barriers in publishing demands a proactive attitude. Nature Human Behaviour, 3(10), 1034.

Vuong, Q.-H. (2020). Reform retractions to make them more transparent. Nature, 582(7811), 149. 